\title{
COMPARING INSPIRE AND OPENSTREETMAP DATA: HOW TO MAKE THE MOST OUT OF THE TWO WORLDS
}

\author{
M. Minghini ${ }^{1, *}$, A. $\operatorname{Kotsev}^{1}$, M. Lutz ${ }^{1}$ \\ ${ }^{1}$ European Commission, Joint Research Centre (JRC), Via Enrico Fermi 2749, 21027 Ispra, Italy \\ (marco.minghini, alexander.kotsev, michael.lutz)@ec.europa.eu
}

Commission IV, WG IV/4

KEY WORDS: Geospatial Data, INSPIRE, Interoperability, OpenStreetMap, Spatial Data Infrastructures, Volunteered Geographic Information

\begin{abstract}
:
The beginning of our century has seen the rise of Spatial Data Infrastructures (SDIs) and crowdsourced geographic information projects. This study analyses and compares the most relevant initiatives for Europe in both contexts: INSPIRE, the Directive aiming to establish a pan-European SDI used for environmental policies, and OpenStreetMap (OSM), the largest and richest crowdsourced geospatial database. Similarities and differences, advantages and disadvantages of the two initiatives from an end user perspective are presented for a number of characteristics: underlying approach and governance, spatial scope, data structure and encoding, data access, and licensing framework. Overall, both initiatives have developed specific strengths and have achieved different types and degrees of interoperability, which would make their integration highly beneficial to multiple stakeholders. From the pure technical perspective, such integration is fully enabled by the maturity of the available FOSS4G, which offers specific support for both INSPIRE and OSM resources, also reviewed in the paper.
\end{abstract}

\section{INTRODUCTION}

The way geospatial information is produced, distributed, accessed and exchanged has witnessed dramatic changes in the last few decades. Until the end of the last century, the activities of mapping and geospatial data collection have solely belonged to the realm of professionals, while data ownership and sharing have been for decades the only prerogative of national mapping agencies. Curiously, the same year (1994) that saw the light of the first, rudimentary application able to display geographic content on the Web (Putz, 1994) was also the year when the related concept of Spatial Data Infrastructure (SDI) was first theorised in the US as 'the technology, policies, standards, and human resources necessary to acquire, process, store, distribute, and improve utilization of geospatial data' (Clinton, 1994). Since then, and particularly at the beginning of the new century with the rise of the first Open Geospatial Consortium (OGC) standards for geospatial interoperability, SDIs have begun to appear in practice at all scales, from the local and regional up to the national and international (Peng, Tsou, 2003). Still today, core components of SDIs - in addition to interoperability standards - are policies, partnerships with relevant communities, geospatial data, metadata, services, web catalogues and geoportals.

Soon after the growth of the first SDIs, driven by governments and mapping agencies, a number of significant technological advances including Web 2.0, proliferation of location-enabled mobile devices, open access to satellite imagery and online maps, have enabled citizens to become key players in the context of production and sharing of geospatial information (Foody et al., 2017). This phenomenon is traditionally known as Volunteered Geographic Information (VGI) (Goodchild, 2007), although a multitude of similar terms were coined in literature which are overall captured by the general concept of crowdsourced geographic information (See et al., 2016). The number

\footnotetext{
${ }^{*}$ Corresponding author
}

of such initiatives, continually growing thanks to the emerging opportunities offered by current technology, spans a large number of purposes, disciplines, communities and tools, with an enormous body of literature already produced.

The present work fits in this context by analysing and comparing the most relevant initiatives for Europe related to SDIs and VGI, i.e. INSPIRE and OpenStreetMap, respectively. INSPIRE (Infrastructure for Spatial Information in Europe, https://inspire.ec.europa.eu) is the Directive providing a legal, technological and organisational framework for the creation of a single pan-European SDI for the purposes of environmental policies based on the infrastructures already established and operated by European Union (EU) Member States (MS) (European Commission, 2007). The Directive entered into force in 2007 with a roadmap setting out target dates for different implementation stages, with full implementation required for 2021. Initiated in response to the evidence of the huge amount of barriers preventing the sharing and re-use of geospatial data in Europe, INSPIRE represents the largest effort in building an SDI to date, and thus represents a reference for current and future similar initiatives (Cetl et al., 2019). Details on the organisational and technical characteristics of INSPIRE are provided in Section 2.

In parallel, OpenStreetMap (OSM, https://openstreetmap.org) is the most successful VGI project to date. Started in 2004 in response to the limited or no availability of openly-licensed authoritative maps, the project aims to create a crowdsourced geospatial database of the whole world utilisable under an open access license (see Subsection 2.4.1 in the following). A key ingredient for the popularity of OSM is that anyone can contribute despite not having specific skills in cartography or geoinformatics. At the time of writing (June 2019) the number of users registered to OSM is about 5.5 million, while the number of contributors (i.e. users who have made at least one edit to the 
database) is just over 1 million (https://osmstats.neis-one.org). The global extent, richness and level of detail of the OSM database have attracted a high academic interest (Jokar Arsanjani et al., 2015) as well as an increasing exploitation by a number of actors to build a complex infrastructure of services and applications (Mooney, Minghini, 2017). Because of this, OSM can be considered a crowdsourced SDI. The most relevant features of the OSM project are described in more detail in Section 2.

The remainder of the paper is organised as follows: Section 2 represents the core of the work, providing a one-to-one comparison between INSPIRE and OSM on several specific aspects, ranging from the technical to the legal and organisational ones, with the goal of identifying similarities and differences and highlighting pros and cons of the two initiatives. Section 3 provides an overview of the most popular open source software solutions providing specific support for INSPIRE and OSM resources. Section 4 concludes the paper by discussing the outcomes of the INSPIRE-OSM comparison in the broader context of the integration between SDI and VGI data, highlighting the opportunities to make the most out of them, as well as the associated challenges.

\section{INSPIRE-OPENSTREETMAP COMPARISON}

Table 1 provides a synthesised comparison between INSPIRE and OSM for a number of characteristics, starting from the managerial and organisational ones, and then diving into more technical aspects about the data produced by the two initiatives. For each analysed characteristic, the similarities and differences are elaborated in further detail in the following subsections.

\begin{tabular}{|c|c|c|}
\hline Characteristic & INSPIRE & OpenStreetMap \\
\hline Approach & $\begin{array}{c}\text { top-down } \\
\text { Spatial scope }\end{array}$ & $\begin{array}{c}\text { bottom-up } \\
\text { spatial data themes } \\
\text { any spatial object } \\
\text { (verifiable) }\end{array}$ \\
$\begin{array}{c}\text { Data structure } \\
\text { and encoding } \\
\text { complex data model, } \\
\text { GML encoding }\end{array}$ & $\begin{array}{c}\text { flat data model, GDAL } \\
\text { supported formats }\end{array}$ \\
Data CRS & $\begin{array}{c}\text { INSPIRE-specific } \\
\text { CRSs }\end{array}$ & WGS84 \\
Data access & $\begin{array}{c}\text { OGC-compliant } \\
\text { clients, Geoportal } \\
\text { different, depending } \\
\text { on MS data providers }\end{array}$ & $\begin{array}{c}\text { APIs, Planet File, } \\
\text { predefined extracts }\end{array}$ \\
Data license & ODbL \\
\hline
\end{tabular}

Table 1. Synthesised comparison between INSPIRE and OSM

\subsection{Approach}

The main difference between INSPIRE and OSM is their underlying approach. Coordinated by the European Commission (EC) and the European Environment Agency (EEA), INSPIRE has been conceived in a top-down direction, since the common Implementing Rules required by the Directive - adopted as Commission Decisions or Regulations and covering the core components of the infrastructure - are legally binding for public authorities in the EU MS. In other words, they must implement the INSPIRE legal requirements by the target dates specified in the roadmap (https://inspire.ec.europa.eu/inspireroadmap). Since the INSPIRE Implementing Rules are EU legislation, their implementation can be enforced and noncompliance might ultimately lead to infringement procedures. However, at the same time, since its birth INSPIRE has been implemented as a highly participatory initiative. In fact, the development of the INSPIRE legal and technical documents and of the maintenance and implementation framework were based on an open and inclusive process, involving experts from the stakeholder community in the MS. MS representatives have also an important role in the INSPIRE governance structure (https://inspire.ec.europa.eu/whos-whoinspire/57734), for example within the Maintenance and Implementation Group (MIG). In addition, experts from the INSPIRE community can discuss implementation issues on the Community Forum (https://inspire.ec.europa.eu/forum) as well as through helpdesk channels dedicated to specific implementation tools, e.g. those for the INSPIRE Geoportal and the INSPIRE Reference Validator (https://github.com/inspire-euvalidation/community). These discussions are closely monitored by the INSPIRE technical and political coordinators and sometimes lead to agreed changes to the official legal and/or technical documentation. Finally, the INSPIRE Conference is the annual event gathering the INSPIRE community, formed by MS representatives, data providers and INSPIRE implementers, companies providing technical support for INSPIRE, stakeholders and users, and EC and EEA staff (https://inspire.ec.europa.eu/portfolio/inspire-conferences).

OSM has developed in an opposite, bottom-up direction. The very idea of the project, initiated in 2004 by then M.Sc. student Steve Coast, was to crowdsource the mapping of the whole world through the contributions of a large number of users, each having local knowledge on a specific area (Mooney, Minghini, 2017). As a consequence, over time the OSM database has grown through a fully spontaneous process, largely driven by the enthusiasm of volunteers willing to put their time and effort in creating an openly-licensed product from which everyone can benefit (see Subsection 2.4). OSM is supported, but not controlled, by the OpenStreetMap Foundation (OSMF), a not-for-profit organisation which provides legal support to the project, maintains its server infrastructure, and promotes fund-raising to ensure its sustainability (https://wiki.osmfoundation.org/wiki/Main_Page). The OSMF has its own governance structure, mainly composed of a Board and a number of Working Groups supporting OSM in specific areas (licensing issues, vandalism, communication, etc.). Despite the presence of the OSMF, OSM contributors are the only owners of the database. Similarly to the case of INSPIRE, the OSM community also meets annually in a global event named 'State of the Map', which attracts users and developers as well as public administrations, companies and researchers working with OSM data (https://wiki.openstreetmap.org/wiki/State_Of_The_Map).

\subsection{Spatial scope}

The goal of INSPIRE is the creation of a European-wide SDI for the purposes of European environmental policies, and policies or activities which may have an impact on the environment. As such, the Directive addresses 34 so-called spatial data themes relevant for environmental applications, which are listed and defined in the Annexes of the Directive (European Commission, 2007). The themes of Annex I and partly Annex II define a spatial reference framework the remaining themes refer to (see Figure 1). For each theme, the INSPIRE data models (see Subsection 2.3) define and rigorously document on a conceptual level one or more spatial data object types to be used for sharing the data. INSPIRE themes include a total of about 340 spatial object types (http://inspireregadmin.jrc.ec.europa.eu/dataspecification/CatalogueINSPIRE 
Objects.action). In a nutshell, INSPIRE data pertains to very specific geospatial domains (e.g. transportation, statistics, ecology, meteorology, oceanography). While more general and non-environmental geospatial datasets (such as points of interest) are not explicitly included in the spatial scope of INSPIRE, they can still be extracted from multiple specific themes.

\begin{tabular}{|c|c|c|}
\hline Annex I & Annex III & \\
\hline 1. Coordinate reference systems & 1. Statistical units & 11. Area management/ \\
\hline 2. Geographical grid systems & 2. Buildings & ation zones \\
\hline 3. Geographical names & 3. Soil & \\
\hline 4. Administrative units & 4. Land use & 12. Natural risk zones \\
\hline 5. Addresses & 5. Human health and safety & 13. Atmospheric conditions \\
\hline 6. Cadastral parcels & 6. Utility and governmental & $\begin{array}{l}\text { 14. Meteorological } \\
\text { geographical features }\end{array}$ \\
\hline 7. Transport networks & 7. Environmentalmonitoring & 15. Oceanographic \\
\hline 9. Protected sites & facilities & $\begin{array}{l}\text { geographical features } \\
\text { Searegions }\end{array}$ \\
\hline Annex II & facilities & 17. Bio-geographical regions \\
\hline 1. Elevation & $\begin{array}{l}\text { 9. Agricultural and aquaculture } \\
\text { facilities }\end{array}$ & $\begin{array}{l}\text { 18. Habitats and biotopes } \\
\text { 19. Species distribution }\end{array}$ \\
\hline 2. Land cover & 10.Population distribution - & 20. Energy Resources \\
\hline 3. Ortho-ima & demography & 21. Mineral resources \\
\hline 4. Geology & & \\
\hline
\end{tabular}

Figure 1. INSPIRE spatial data themes, divided in three Annexes (source: European Commission, 2007)

Conversely, OSM was started with the goal of producing a database of streets (hence the name 'OpenStreetMap') but did soon evolve into the most diverse geospatial database available. In the open spirit of the project, any object having a physical location on the Earth surface and being verifiable, i.e. provable to be true or false (https://wiki.openstreetmap.org/wiki/Verifiability), can be added (at any time and by any contributor) in the OSM database. Consequently, a documented list of all OSM objects has been produced and agreed upon over time by the community. The list is maintained on a dedicated Map Features wiki page (https://wiki.openstreetmap.org/wiki/Map_Features), which evolves dynamically as new object types are created. This happens through a collaborative procedure, i.e. the proposal to add a new object type is presented (by properly justifying its need and impact) and the OSM community openly votes for acceptance or rejection. The result is a highly diversified list of several hundreds of object types (including indoor object types) pertaining to almost any geospatial domain. Thus, compared to INSPIRE, the spatial scope of OSM is in general wider, but - as a consequence of the verifiability principle the database does not include historic events (such as environmental observations) and objects that do not exist anymore in the real world.

\subsection{Data structure and encoding}

One of the greatest differences between INSPIRE and OSM concerns the way to model and encode data. For each of the 34 spatial data themes, INSPIRE data models have been originally defined through the involvement of a large number of stakeholders from MS as well as domain-specific experts. From an interoperability perspective, the overall goal was to define models that are sufficiently articulated and capture the peculiar characteristics to be used in all European Union MS to refer to the same types of real-world geospatial entities (for example, the many different ways used in MS to define addresses or protected sites).

INSPIRE conceptual models for all spatial data themes are defined using the Unified Modelling Language (UML). These models, accessible online from a common UML repository (https://inspire.ec.europa.eu/Data-Models/DataSpecifications/2892), represent the foundation of the INSPIRE
Implementing Rules and the corresponding data specification Technical Guidance documents, the latter specifying the technical approaches that MS can adopt in order to satisfy the legal obligations of the Implementing Rules. The INSPIRE UML models have been created based on a number of European use cases in each particular domain. This, together with the specific modelling approach adopted, has resulted into a general sophistication. By way of example, models include complex (i.e. non-simple) attributes and data types, properties with multiplicity greater than 1 , and a wide range of available geometry types (including mixed geometries). The INSPIRE Implementing Rules on the interoperability of spatial data sets and services allow the use of any encoding rule which conforms to EN ISO 19118 (International Organization for Standardization, 2011), specifies schema conversion rules for all spatial object types and all attributes and association roles and the output data structure used, and is publicly made available (European Commission, 2010). However, the default encoding rule for all INSPIRE data themes maps INSPIRE UML models into Geography Markup Language (GML) application schemas (XML schemas). They are made available in the INSPIRE schema repository (https://inspire.ec.europa.eu/schemas). Compliance of GML datasets against the requirements of the Technical Guidance documents are tested through the INSPIRE Validator (https://inspire.ec.europa.eu/validator). Recently, an alternative rule has been developed by the INSPIRE MIG (https://github.com/INSPIRE-MIF/2017.2) proposing an encoding of INSPIRE data that departs from the same UML conceptual model and is based on the GeoJSON standard (Butler et al., 2016).

OSM's conceptual data model of the physical world is simpler. Any OSM object is merely described through the combination of an element (specifying the object geometry) and a list of one or more tags (defining the object attributes) (Ramm et al., 2010). OSM elements can be of three types: nodes, used to represent standalone point features and defined by a latitude and a longitude; ways, i.e. ordered lists of between 2 and 2,000 nodes which represent both linear and polygon features; and relations, i.e. multi-purpose data structures documenting relationships between two or more elements (nodes, ways, and/or other relations) (https://wiki.openstreetmap.org/wiki/Elements). Tags, consisting of simple key/value pairs, are associated to each OSM element to describe its properties (https://wiki.openstreetmap.org/wiki/Tags). In the open spirit of the OSM project, the Map Features wiki page (https://wiki.openstreetmap.org/wiki/Map_Features) as well as all the wiki pages reachable from it, lists the recommended tags agreed by the community, but - in contrast to INSPIRE, where models have been created based on a number of European use cases - OSM contributors are in principle free to define and use their own tags. For example, it may happen that national OSM communities agree to introduce additional tags to describe specific properties of national or local importance. Regardless of this, the fundamental difference when compared to INSPIRE is the flat OSM data structure, which allows to encode OSM data in any available vector format supported by e.g. the Geospatial Data Abstraction Library (GDAL, https://gdal.org) without any loss of information. As discussed later in Section 3 , this ensures a wide client support for consuming OSM data. However, the original OSM data format (provided e.g. by the OSM API, see Subsection 2.4) is XML-based.

2.3.1 Coordinate Reference System The Coordinate Reference System (CRS) in which INSPIRE and OSM data are 
provided deserves a separate discussion. To ensure interoperability, INSPIRE mandates the use of specific, pan-European CRSs, e.g. using geodetic coordinates based on the ETRS89 or the ITRS datum, or plane coordinates based on the ETRS89 datum and the Lambert Azimuthal Equal Area, Lambert Conformal Conic, or Transverse Mercator projections. Common three-dimensional CRSs (Cartesian and geodetic) are also defined (European Commission, 2010). However, since in many cases the effect of this requirement is that MS have to create, store and maintain data in both their national CRS and one of the INSPIRE-required CRSs, or to use the Download and View Services to provide the required CRSs, the INSPIRE expert group is currently discussing a mechanism that would make it easier to allow additional CRS in order to lower the burden for implementers. In such a case, CRS transformations would need to be implemented using available tools, or libraries such as GDAL and PROJ (https://proj.org). OSM data are instead provided in the WGS84 CRS (with no three-dimensional component), the reason being the use of GPS devices to collect street data when the project was originally started. Since OSM editors (based on the OSM API, see Subsection 2.4) only allow contributors to add OSM data in WGS84, this already ensures full CRS compatibility for the whole database.

\subsection{Data access}

As a world-class example of an SDI, INSPIRE is based on the set of core components mentioned in Section 1. A key role in the infrastructure is played by metadata, based on the established EN ISO 19115 and EN ISO 19119 standards and allowing to find the data published by European Union MS described in Subsections 2.2 and 2.3. Both data and metadata are shared through a Service-Oriented Architecture (SOA) approach, where the so-called INSPIRE Network Services are setup based on OGC standards: 'Discovery Services', to establish access to metadata through the Catalogue Service for the Web (CSW); 'View Services', to provide interactive data visualisations through the Web Map service (WMS) and Web Map Tile Service (WMTS); and 'Download Services', to offer download of raw data through the Atom Syndication Format (Nottingham, Sayre, 2005), the Web Feature Service (WFS), the Web Coverage Service (WCS), or the Sensor Observation Service (SOS). Similarly to the case of data, the compliance of metadata and services to the INSPIRE Technical Guidance documents is tested through the INSPIRE Validator (https://inspire.ec.europa.eu/validator).

Any OGC-compliant client application implementing those standards is thus able to access data and metadata exposed by MS (see also Section 3 in the following). However, another core component of the INSPIRE SDI is the Geoportal (http://inspiregeoportal.ec.europa.eu), acting as the main client application of the whole infrastructure and providing a central point of access to the whole set of services from MS organisations. The INSPIRE Geoportal does not store geospatial data. Instead, it exposes data through harvesting the CSW endpoints made available by MS. In addition to data access, for each MS the Geoportal provides statistics on the number of available resources: metadata records, datasets available through View Services, and datasets available through Download Services (see Figure 2, corresponding to the situation as of June 7, 2019). As demonstrated by the difference between the number of metadata records and the number of downloadable datasets (both for the single MS and as a whole), full implementation of INSPIRE has not yet been achieved. This is also proven by the fact that
MS datasets published in the Geoportal include both datasets compliant to the INSPIRE data models (see Subsection 2.3) as well as as-is (i.e. non compliant) datasets. Selecting a MS or an INSPIRE theme, the Geoportal allows users to browse available data (through their metadata), view and download them.

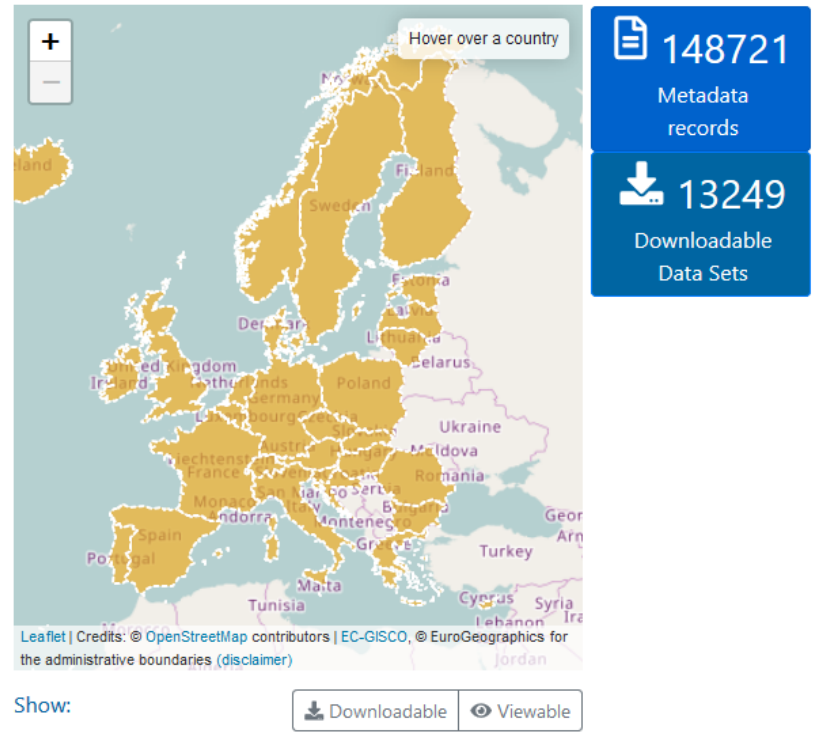

INSPIRE Geoportal Data Set Statistics

\begin{tabular}{|c|c|c|c|}
\hline \\
\hline Eustria & 目 $554 \mid$ 上 $403 \mid$ ○ 417 & Eatvia & 目 $139|\mathbf{\perp} 10| \boldsymbol{O} 20$ \\
\hline D| Belgium & 国 571 | & Liechtenstein & 영 $59| \pm 9| \odot 11$ \\
\hline Bulgaria & 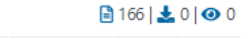 & Lithuania & 司 $12 \mid$ 丸 $10 \mid \odot 11$ \\
\hline Droatia & 目 $115|\mathbf{\perp} 7| \odot 7$ & ELuxembourg & 目 $197 \mid \mathbf{\Delta} 192$ | 163 \\
\hline Cyprus & 固42|丸27|○0 & ${ }^{+}$Malta & 目157|」 $129 \mid$ ○ 151 \\
\hline Czech Republic & 司 $149 \mid$ 丸 $38 \mid \bigcirc 95$ & Eetherlands & 固 $199|\mathbf{\perp} 122|$ ○ 129 \\
\hline Penmark & 目223|上28|②7 & 揊 Norway & 国205|⿺ $33 \mid$ ○ 10 \\
\hline Estonia & 目 $75 \mid$ 上 $14 \mid \odot 23$ & - Poland & 目 $32286 \mid \boldsymbol{\perp} 103$ | ○ 14 \\
\hline t Finland & 包555|さ5 $50 \mid \odot 52$ & (c) Portugal & 直 $610|\boldsymbol{\perp} 104|$ ○ 206 \\
\hline DFrance & 国 39328 | 上 845 | O 10118 & IRomania & 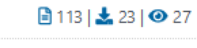 \\
\hline Germany & 国 30484 | 上 $10616 \mid$ O 10798 & (O-) Slovakia & 国238|上 $18 \mid$ ○ 18 \\
\hline 琵Greece & 固57|む2| 57 & Slovenia & 目 $87 \mid$ 土 $15 \mid$ ○ 8 \\
\hline EHungary & 비일 & Ispain & 固219|」 $142 \mid \odot 150$ \\
\hline 믐 Iceland & 国 $147|\downarrow 7| \odot 0$ & IE sweden & 国295|む27|⑫0 \\
\hline IIreland & 国 $53| \pm 0| \odot 0$ & † switzerland & 目208|上2|厂0 \\
\hline I Italy & 国20278|山0| 385 & 柴区 United Kingdom & 国 $20787 \mid$ ג $63 \mid$ | 165 \\
\hline
\end{tabular}

Figure 2. Availability of metadata, viewable and downloadable datasets on the INSPIRE Geoportal

Data access happens in a totally different fashion for OSM. Thanks to the relative simplicity of its conceptual model, OSM data can be easily accessed from a variety of sources and in a variety of formats. Metadata catalogues are also not needed, since metadata information is in a large part already included in the tags of OSM objects, and data search/access is only based on tags. The easiest way to download data is through the OSM website (https://openstreetmap.org), selecting the $E x$ port functionality and defining the bounding box of interest. Application Programming Interfaces (APIs) are also available, which offer programmatic data access to the OSM database. The OSM API (https://wiki.openstreetmap.org/wiki/API), used 
by OSM editors (https://wiki.openstreetmap.org/wiki/Editors), provides read and write access to the database, while the Overpass API (https://wiki.openstreetmap.org/wiki/Overpass_API), mostly used from the popular web front-end Overpass Turbo (https://overpass-turbo.eu), provides read-only access with customised query capabilities, which makes it ideal for data download. One of the peculiar characteristics of OSM is the availability - together with the database - of its history, which includes the whole set of edits performed on each OSM object and represents an extremely interesting data source for researchers, e.g. to study OSM spatio-temporal evolution (Minghini et al., 2018). The ohsome platform (https://ohsome.org) was recently developed to provide API-based access to the OSM history.

Another popular way to access OSM data is through the Planet OSM (https://planet.openstreetmap.org), a weekly-updated file including the global OSM database. Along with the Full History Planet OSM, i.e. the version which also includes the whole OSM history (https://planet.openstreetmap.org/planet/fullhistory), it is available in the standard XML format as well as the Protocolbuffer Binary Format (PBF). Finally, a number of companies and organisations offer predefined OSM extracts available for download. These are obtained from a pre-processing of the OSM database to e.g. cover specific areas and/or include only specific objects (buildings, road networks, land use features, etc.) and are offered in multiple formats and CRSs. Examples are the OSM data extracts provided by Geofabrik (http://download.geofabrik.de), Interline (https://www.interline.io/osm/extracts), and the HSR University of Applied Sciences (https://osmaxx.hsr.ch) as well as those that can be dynamically generated through the Humanitarian OpenStreetMap Team (HOT) Export Tool (https://export.hotosm.org/en/v3).

2.4.1 Data license Licensing constitutes another fundamental point of difference in the INSPIRE and OSM data sharing approaches. The INSPIRE Directive does not provide any obligation on the license under which MS data shall be made available. As a consequence, the infrastructure has developed in a very heterogeneous way in terms of data licenses. Many datasets are published under open access licenses, while many others are missing license information, or are subject to different, and sometimes restrictive, conditions on their access and use. Both standard licenses (e.g. belonging to the CC BY family) and customised licenses (often provided only in national languages) are specified in the dataset metadata. In addition, INSPIRE allows MS to restrict the view and download of datasets under certain conditions, e.g. if access to those datasets might adversely affect public security or national defence (European Commission, 2007). The result is a heterogeneous picture, which sometimes makes it difficult for end users to understand which legal conditions apply to the use of datasets obtained from combining two or more INSPIRE datasets. In contrast, the whole OSM database is available under a single open access license, the Open Database License (ODbL) (Open Data Commons, 2019). This license allows everyone to freely copy, distribute, transmit and adapt the data, as long as credit is made to OSM and its contributors; also, when altering or building upon the OSM database, the result shall be distributed under the same licence (https://www.openstreetmap.org/copyright).

\section{FOSS4G FOR INSPIRE AND OSM}

A multitude of software tools can be used in order to search, access, visualise, analyse and process INSPIRE and OSM data.
Clearly, considering the fact that both OSM and INSPIRE data are geospatial by nature, there is a certain overlap between the tools for their creation, maintenance and consumption. There are however some noteworthy differences. Firstly, while INSPIRE is supported by both proprietary and open source software solutions, because of its very nature OSM has mostly stimulated the development of new open source software. As a contribution to the conference where this work is presented, the following discussion focuses on the most popular Free and Open Source Software for Geospatial applications (FOSS4G) providing specific support for INSPIRE and OSM resources. In other words, the discussion only concerns software (or parts thereof) which is specifically developed to address the peculiar characteristics of INSPIRE and OSM described in Section 2.

An inventory of tools useful for INSPIRE implementation is available at https://inspire-reference.jrc.ec.europa.eu/tools. These tools include both proprietary and open source solutions ranging from desktop/server software, libraries, plugins, online services and other technical products suitable to share and consume INSPIRE data, metadata and services. Only relevant FOSS4G tools are described in the following. Regarding data discovery, specific support for INSPIRE is provided by GeoNetwork opensource (https://geonetwork-opensource.org), used by more than half of the European Union MS to setup their national catalogues; pycsw (https://pycsw.org), an implementation of CSW written in Python and implementing INSPIRE Discovery Services; and deegree (https://www.deegree.org), which comes with an INSPIRE workspace to help providing the services required by INSPIRE. Deployment of INSPIRE services for data visualisation and download can be achieved through a number of FOSS4G solutions. GeoServer (http://geoserver.org) provides an INSPIRE extension (https://docs.geoserver.org/stable/en/user/extensions/inspire) offering INSPIRE-specific configuration for WMS, WMTS, WFS and WCS capabilities documents. Another GeoServer extension is the application schema support (appschema, https://docs.geoserver.org/maintain/en/user/data/appschema), which offers WFS support for complex feature types conforming to a GML application schema. MapServer (https://mapserver.org) allows also to deploy INSPIRE-compliant View Services (https://mapserver.org/ogc/inspire.html) and Download Services (https://www.mapserver.org/ogc/inspire_dl.html). Another well-used web geospatial server is deegree, which, in addition to CSW, provides also an INSPIRE-compliant implementation of WMS, WMTS, WFS and WCS. Instead, the most successful open source product to serve INSPIRE-compliant spatio-temporal observation data from sensors is $52^{\circ}$ North SOS (https://52north.org/software/software-projects/sos). INSPIRE services and geoportals can be also created using the Mapbender framework (https://www.mapbender.org), which is especially used in Germany, as well as GeoNode (http://geonode.org), a well-known web-based platform used to deploy SDIs which is built with pycsw embedded as default CSW component (with GeoNetwork opensource and deegree configurable as alternate CSW servers) and GeoServer as default OGC web services (OWS) component.

As demonstrated in an ongoing study on INSPIRE client support (https://github.com/INSPIRE-MIF/caniuse), the most popular open source geospatial web clients OpenLayers (https://openlayers.org) and Leaflet (https://leafletjs.com) provide no support for INSPIRE GML data. This is among the reasons resulted into the recent activity of the INSPIRE 
MIG on creating an INSPIRE UML-to-GeoJSON encoding rule (as mentioned in Subsection 2.3), since the GeoJSON format is especially suitable for data consumption on the Web. Regarding desktop clients, the FOSS4G tool providing the highest support for INSPIRE GML data is QGIS (https://qgis.org). It offers a number of ad hoc plugins to enable the full consumption of INSPIRE data: for QGIS 3+, the most powerful one is 'GML Application Schema Toolbox' (https://github.com/BRGM/gml_application_schema_toolbox), explicitly developed to allow manipulating GML application schema datasets in QGIS. Several plugins allow instead to directly query and add in QGIS the INSPIRE datasets published by MS, e.g. the 'INSPIRE Nederland plugin voor QGIS' (https://plugins.qgis.org/plugins/inspireNL). In addition, GRASS GIS (https://grass.osgeo.org) offers a metadata editor to create and edit metadata compliant to the INSPIRE profile. Finally, ETL (Extract, Transform, Load) open source solutions allow INSPIRE data providers to map and transform their native datasets into data that validates against the INSPIRE schemas. This is assisted by the presence of ready-to-use mapping tables for all INSPIRE themes, available at https://inspire.ec.europa.eu/DataModels/Data-Specifications. The de facto standard, which is most used by European data providers, is hale Studio (https://www.wetransform.to/products/halestudio); an alternative is GeoKettle (http://www.spatialytics.org/projects/geokettle).

In the case of OSM, due to the open-access availability of the database and the programmatic way to access it provided by existing APIs (see Subsection 2.4), the number of available software tools is huge. A comprehensive review of the most popular OSM-based applications for data editing, data download, visualisation, routing and quality assurance was recently compiled (Mooney, Minghini, 2017). There is usually no need for FOSS4G to provide specific OSM support, since the flat and simple structure of OSM data (described in Subsection 2.3) allows open source desktop, web-based and mobile tools to natively load, visualise and process them. Customised tools are mostly available for GIS client applications. For example, OSM data can be loaded in QGIS using specific plugins such as 'QuickOSM' (https://plugins.qgis.org/plugins/QuickOSM) and 'OSMDownloader' (https://plugins.qgis.org/plugins/OSMDownloader), both based on the Overpass API. Instead, OSM basemaps can be loaded using the plugins 'QuickMapServices' (https://plugins.qgis.org/plugins/quick_map_services) or 'OpenLayers' (https://plugins.qgis.org/plugins/openlayers_plugin). Orfeo Toolbox (https://www.orfeo-toolbox.org), an open source software dedicated to remote sensing, provides a specific application named 'OSMDownloader' to download OSM data from the main server and use them as reference data to train classification models (http://tiny.cc/z0j27y). Similar support for OSM is also provided by the desktop software GRASS GIS, in particular on importing OSM data and correcting the topology (https://grasswiki.osgeo.org/wiki/OpenStreetMap), and gvSIG, in particular on loading a number of OSM basemaps (https://blog.gvsig.org/2019/02/28/towards-gvsig2-5-new-osm-map-servers). Similarly to the desktop case, OSM basemaps can be also embedded in web maps - usually as Tile Map Service (TMS) layers - using OpenLayers and Leaflet. Tiles are retrieved either from the OSM servers or from third-party providers which created their own thematic visualisations. A full list of OSM-based visualisation services is available at https://wiki.openstreetmap.org/wiki/List_of_OSMbased_services.

\section{DISCUSSION AND CONCLUSIONS}

SDI and VGI initiatives have existed for many years and the potential for their convergence was recognised since the very beginning (Craglia, 2007). Free availability, amount, richness and up-to-dateness have been traditionally considered as key elements for VGI to beneficially integrate or complement authoritative data collected and managed by national mapping agencies (Olteanu-Raimond et al., 2017). In the specific case of INSPIRE, a number of efforts have been made to integrate its authoritative, standardised data with VGI, but these usually addressed specific case studies without the attempt to conceptualise an integrated framework (Wiemann, Bernard, 2014, Ježek et al., 2015, Charvát et al., 2018). Due to several technical, institutional and legal barriers, this endeavour is still at an early stage and its success calls for different approaches such as the creation of integrated GIS platforms involving a wide network of stakeholders (Demetriou et al., 2017).

This paper analysed the specific example of OSM, the most mature VGI project herewith considered as a crowdsourced SDI, and its comparison with INSPIRE. There is no doubt that the combination of geospatial information extracted from the two initiatives would be significantly beneficial to several stakeholders: public authorities, professionals, businesses, researchers, humanitarian organisations, and the same INSPIRE and OSM communities in a broad sense. The comparison performed in Section 2 outlined the fundamental underlying differences in the two approaches: the rigorous one adopted by INSPIRE, driven by legal obligations and founded on strict data specifications; and the open one characterizing OSM, driven by the freedom left to its contributors.

Taken separately, each of the two projects has achieved different types and degrees of interoperability at the expense of different drawbacks. INSPIRE has been making an impressive investment in harmonising the way geospatial data is modelled and distributed at the pan-European level, establishing a legal, organisational and technical reference for current and future SDI initiatives. This comes at the cost of an overall heterogeneous and slow implementation by MS due to several reasons, e.g. technical complexity, lack of resources and legal/organisational issues at the MS national level. This means that the full implementation of INSPIRE, and the related immense political and managerial benefits it could bring at the European level, is still to be achieved. In addition, two major issues which might prevent the general usability of the INSPIRE infrastructure are the use of a technologically old architecture to share and access data and the heterogeneity of MS data licenses (see Subsection 2.4). On the other side, being an international project since its beginning, OSM has full license interoperability and is founded on modern technologies (mainly APIs) which facilitate not only accessing data but also building third-party applications on top of them. However, by its very flexible nature OSM suffers from the lack of rigorous data specifications, since contributors are free to use tags different from those agreed by the community. In this regard, efforts have been recently made to guide the implementation of VGI projects using data collection protocols (Minghini et al., 2017). Intrinsic cons of VGI have also to be considered, e.g. a typically uneven spatial coverage and the lack of quality assurance, although many literature studies have shown OSM to be of comparable or even better quality than authoritative data.

From the pure technical aspect, Section 3 provided an overview of how the available FOSS4G ecosystem provides specific sup- 
port for INSPIRE and OSM data, demonstrating its overall maturity (Brovelli et al., 2017). Either using OGC web services, APIs or external files, INSPIRE and OSM data can be seamlessly searched and loaded in client applications, processed together to create new content, and also converted to align their data structures. In the latter case, a transformation process is needed in order to either convert OSM data to the INSPIRE schemas, or flatten the INSPIRE models to align them with the simple key/value pair structure of OSM required for imports (https://wiki.openstreetmap.org/wiki/Import/Guidelines). Nevertheless, the cases were INSPIRE and OSM data have been used together are isolated for multiple reasons such as licensing requirements, lack of awareness and data security considerations. Still, they provide an interesting setting and facilitate the use of the data by benefiting from the advantages of both OSM and INSPIRE. Thus, combining INSPIRE and OSM data ultimately requires a comprehensive understanding not only of technical aspects, but also of the processes underlying the creation and maintenance of the two infrastructures. Despite INSPIRE and OSM were born for different purposes and aim to achieve different goals, each of them has developed solid and well-recognised good practices the other can benefit from. Hence, to establish a real, sustainable and extensible integration between the two, an additional effort involving all stakeholders (project leaders, implementers, data providers/contributors and end users) constitutes the necessary step.

\section{DISCLAIMER}

The views expressed are purely those of the authors and may not in any circumstances be regarded as stating an official position of the European Commission.

\section{REFERENCES}

Brovelli, M. A., Minghini, M., Moreno-Sanchez, R., Oliveira, R., 2017. Free and open source software for geospatial applications (FOSS4G) to support Future Earth. International Journal of Digital Earth, 10(4), 386-404. doi.org/10.1080/17538947.2016.1196505.

Butler, H., Daly, M., Doyle, A., Gillies, S., Hagen, S., Schaub, T., 2016. The GeoJSON Format. IETF RFC 7946.

Cetl, V., Tomas, R., Kotsev, A., de Lima, V. N., Smith, R. S., Jobst, M., 2019. Establishing Common Ground Through INSPIRE: The Legally-Driven European Spatial Data Infrastructure. Service-Oriented Mapping, Springer, 63-84.

Charvát, K., Bye, B. L., Mildorf, T., Berre, A. J., Jedlicka, K., 2018. Open Data, VGI and Citizen Observatories INSPIRE Hackathon. International Journal of Spatial Data Infrastructures Research, 13, 109-129. doi.org/10.2902/17250463.2018.13.art11.

Clinton, W., 1994. Coordinating geographic data acquisition and access: the National Spatial Data Infrastructure. Executive Order 12906, Federal Register, 59(71), 17671-674.

Craglia, M., 2007. Volunteered geographic information and spatial data infrastructures: when do parallel lines converge. Position paper for the VGI Specialist Meeting, Santa Barbara, CA, 13-14 December 2007, 13-14.
Demetriou, D., Campagna, M., Racetin, I., Konecny, M., 2017. Integrating Spatial Data Infrastructures (SDIs) with Volunteered Geographic Information (VGI) for creating a Global GIS platform. Mapping and the Citizen Sensor, Ubiquity Press, 273-297.

European Commission, 2007. Directive 2007/2/EC of the European Parliament and of the Council of 14 March 2007 establishing an Infrastructure for Spatial Information in the European Community (INSPIRE). Official Journal of the European Union, L 108/1.

European Commission, 2010. Commission Regulation (EU) No 1089/2010 of 23 November 2010 implementing Directive 2007/2/EC of the European Parliament and of the Council as regards interoperability of spatial data sets and services. Official Journal of the European Union, L 323/11.

Foody, G., Fritz, S., Fonte, C. C., Bastin, L., Olteanu-Raimond, A.-M., Mooney, P., See, L., Antoniou, V., Liu, H.-Y., Minghini, M., Vatseva, R., 2017. Mapping and the citizen sensor. Mapping and the Citizen Sensor, Ubiquity Press, 1-12.

Goodchild, M. F., 2007. Citizens as sensors: the world of volunteered geography. GeoJournal, 69(4), 211-221. doi.org/10.1007/s10708-007-9111-y.

International Organization for Standardization, 2011. ISO 19118:2011 Geographic information - Encoding.

Ježek, J., Jedlička, K., Martolos, J., 2015. Visual Analytics of Traffic-Related Open Data and VGI. Proceedings of the 5th International Conference on Information Society and Technology (ICIST 2015), Kopaonik, Serbia, 8-11 March 2015, 13-26.

Jokar Arsanjani, J., Zipf, A., Mooney, P., Helbich, M., 2015. An introduction to OpenStreetMap in Geographic Information Science: Experiences, research, and applications. OpenStreetMap in GIScience, Springer, 1-15.

Minghini, M., Antoniou, V., Fonte, C. C., Estima, J., OlteanuRaimond, A.-M., See, L., Laakso, M., Skopeliti, A., Mooney, P., Jokar Arsanjani, J., Lupia, F., 2017. The relevance of protocols for VGI collection. Mapping and the Citizen Sensor, Ubiquity Press, 223-247.

Minghini, M., Brovelli, M., Frassinelli, F., 2018. An open source approach for the intrinsic assessment of the temporal accuracy, up-to-dateness and lineage of OpenStreetMap. The International Archives of the Photogrammetry, Remote Sensing and Spatial Information Sciences, XLII-4/W8, 147-154. doi.org/10.5194/isprs-archives-XLII-4-W8-147-2018.

Mooney, P., Minghini, M., 2017. A review of OpenStreetMap data. Mapping and the Citizen Sensor, Ubiquity Press, 37-59.

Nottingham, M., Sayre, R., 2005. The Atom Syndication Format. IETF RFC 4287.

Olteanu-Raimond, A.-M., Laakso, M., Antoniou, V., Fonte, C. C., Fonseca, A., Grus, M., Harding, J., Kellenberger, T., Minghini, M., Skopeliti, A., 2017. VGI in National Mapping Agencies: Experiences and Recommendations. Mapping and the Citizen Sensor, Ubiquity Press, 299-326.

Open Data Commons, 2019. Open Database License (ODbL) v1.0. 
Peng, Z.-R., Tsou, M.-H., 2003. Internet GIS: distributed geographic information services for the internet and wireless networks. John Wiley \& Sons.

Putz, S., 1994. Interactive information services using WorldWide Web hypertext. Computer Networks and ISDN Systems, 27(2), 273-280.

Ramm, F., Topf, J., Chilton, S., 2010. OpenStreetMap: Using and Enhancing the Free Map of the World. UIT, Cambridge.

See, L., Mooney, P., Foody, G., Bastin, L., Comber, A., Estima, J., Fritz, S., Kerle, N., Jiang, B., Laakso, M., Liu, H.-Y., Milinski, G., Niki, M., Painho, M., Pdr, A., Olteanu-Raimond, A.-M., Rutzinger, M., 2016. Crowdsourcing, Citizen Science or Volunteered Geographic Information? The Current State of Crowdsourced Geographic Information. ISPRS International Journal of Geo-Information, 5(5), 55. doi.org/10.3390/ijgi5050055.

Wiemann, S., Bernard, L., 2014. Linking crowdsourced observations with INSPIRE. Proceedings of the 17th AGILE International Conference on Geographic Information Science: Connecting a Digital Europe through Location and Place, Castelln, Spain, 36 June 2014. 